\title{
COMMENTARY
}

\section{Prophylactic open abdomen in patients with postoperative intra-abdominal hypertension}

\author{
Panu Mentula* and Ari Leppäniemi \\ See related research by Batacchi et al., http://ccforum.com/content/13/6/R194
}

\begin{abstract}
Postoperative intra-abdominal hypertension (IAH) is a frequent occurrence in critically ill patients operated on for severe abdominal trauma, secondary peritonitis or ruptured abdominal aortic aneurysm. IAH may progress to abdominal compartment syndrome (ACS) with new-onset organ dysfunction. Early recognition of IAH and interventions that prevent the development of ACS may preserve vital organ functions and increase the probability of survival. The best method to prevent postoperative ACS is to leave the abdomen open during the operation. The decision to leave the abdomen open is usually based on the surgeon's judgment without intra-abdominal pressure (IAP) measurements during the operation. Because significant morbidity and mortality are associated with the open abdomen, the measurement of IAP immediately after the fascial closure, when feasible, could offer an objective method for determining the optimal IAP threshold for leaving the abdomen open. The management of the open abdomen requires a temporary abdominal closure (TAC) system that would ideally prevent the development of ACS and facilitate later primary fascia closure. Among several TAC systems, the most promising are those that provide negative pressure to the wound or continuous fascial traction or both.
\end{abstract}

A considerable number of patients with surgical emergencies may develop visceral or retroperitoneal oedema due to severe inflammation, shock and fluid resuscitation. This oedema may prevent primary closure of the abdomen or may lead to dangerously high intra-abdominal

*Correspondence: panu.mentula@hus.fi

Department of Gastroenterological Surgery, Helsinki University Central Hospital, PL 340, 00029 HUS, Finland pressure (IAP) and abdominal compartment syndrome (ACS) if abdominal closure is attempted by force. As reported in the previous issue of Critical Care, Matano and colleagues [1] used the protocol for the open abdomen based on intraoperative IAP measurement after suturing of the fascia. The cutoff value of IAP for the open abdomen was $12 \mathrm{~mm} \mathrm{Hg}$, which was considerably lower than the recommended cutoff value of IAP $(20 \mathrm{~mm} \mathrm{Hg})$ for decompressive laparotomy because of ACS [2]. Using this protocol, the authors showed overall low mortality and high rate of delayed primary fascial closure using either vacuum-assisted closure or the Bogota bag. Although there is evidence that grade I or II (12 to $20 \mathrm{~mm} \mathrm{Hg}$ ) intra-abdominal hypertension (IAH) [2] can disturb renal function [3], the maximal IAP level that patients tolerate without risk of severe adverse effects at the end of the primary operation or after subsequent closure of the open abdomen needs to be defined. In this context, the most relevant question is whether patients with grade I or II IAH after the fascial closure benefit from prophylactic opening of the fascia or not.

Management of the open abdomen with temporary abdominal closure (TAC) takes considerable health care resources and predisposes the patient to the development of complex ventral hernia [4] and intestinal fistulas [5]. Delayed primary fascial closure cannot be achieved in a considerable proportion of patients with the open abdomen, and prolonged management of the open abdomen increases the risk for complications [6]. Factors that affect delayed primary closure rate may involve TAC technique, aetiology of the open abdomen, and the severity of visceral oedema and factors affecting its resolution [6,7]. There are few evidence-based data to support one TAC technique over another. A recent systematic review [7] suggested that vacuum-assisted closure and methods that provide continuous fascial traction result in a higher delayed primary fascial closure rate than other methods. The first randomized trial that compared vacuumassisted closure and polyglactin mesh-assisted closure systems showed equal results in terms of delayed primary fascial closure [8]. 
As shown by Matano and colleagues [1], the vacuumassisted closure technique drains excess peritoneal fluid better than the Bogota bag, resulting in faster decrease of IAP and thus facilitating earlier closure of the abdomen. Another important component in the vacuum-assisted closure technique is a polyurethane nonadherent layer that covers the viscera and prevents the formation of adhesions between the viscera and the peritoneum. This may facilitate a better late closure rate [9] because these adhesions are one of the main reasons why late fascial closure fails in patients with the open abdomen [10]. In cases with prolonged severe visceral swelling, the vacuum-assisted closure system does not prevent lateral retraction of the fascial edges. In such cases, the method that combines vacuum-assisted closure and meshmediated fascial traction has shown an excellent delayed primary closure rate [11].

Mortality in patients with the open abdomen is high but high mortality rates most probably reflect the overall severity of illness in these patients and are not related to the open abdomen itself [12]. However, complications that can occur in the course of open abdomen management may be related to the TAC techniques and are likely to cause some excess mortality [5]. Before more evidencebased data are provided, the prophylactic use of the open abdomen in surgical patients should be preserved for those with high risk of ACS and death. Peroperative IAP measurement after fascial closure may help to predict the risk of ACS and may be useful in cases in which the clinical decision for leaving the abdomen open is not easy.

In conclusion, Matano and colleagues [1] showed clearly that the vacuum-assisted closure outperforms the Bogota bag in the treatment of the open abdomen. The results of the study are convincing and these should encourage everyone to abandon the use of the Bogota bag and switch to vacuum-assisted closure systems in patients with the open abdomen. However, more studies are needed to clarify indications for prophylactic open abdomen in patients with postoperative IAH.

\section{Abbreviations}

ACS = abdominal compartment syndrome; $\mid \mathrm{AH}=$ intra-abdominal hypertension; IAP = intra-abdominal pressure; TAC = temporary abdominal closure.
Competing interests

The authors declare that they have no competing interests.

Published: 4 February 2010

\section{References}

1. Batacchi S, Matano S, Nella A, Zagli G, Bonizzoli M, Pasquini A, Anichini V, Tucci V, Manca G, Ban K, Valeri A, Peris A: Vacuum-assisted closure device enhances recovery of critically ill patients following emergency surgical procedures. Crit Care, 13:R194.

2. Cheatham M, Malbrain ML, Kirkpatrick A, Sugrue M, Parr M, De Waele J, Balogh Z, Leppäniemi A, Olvera C, Ivatury R, D'Amours S, Wendon J, Hillman K, Wilmer A: Results from the International Conference of Experts on Intraabdominal Hypertension and Abdominal Compartment Syndrome. II. Recommendations. Intensive Care Med 2007, 33:951-962.

3. Dalfino L, Tullo L, Donadio I, Malcangi V, Brienza N: Intra-abdominal hypertension and acute renal failure in critically ill patients. Intensive Care Med 2008, 34:707-713.

4. Jernigan TW, Fabian TC, Croce MA, Moore N, Pritchard FE, Minard G, Bee TK: Staged management of giant abdominal wall defects: acute and longterm results. Ann Surg 2003, 238:349-355; discussion 355-357.

5. Fischer PE, Fabian TC, Magnotti LJ, Schroeppel TJ, Bee TK, Maish GO, Savage $S A$, Laing AE, Barker AB, Croce MA: A ten-year review of enterocutaneous fistulas after laparotomy for trauma. J Trauma 2009, 67:924-928.

6. Miller R, Morris J, Diaz J, Herring M, May A: Complications after 344 damagecontrol open celiotomies. J Trauma 2005, 59:1365-1364.

7. Boele van Hensbroek P, Wind J, Dijkgraaf MGW, Busch ORC, Goslings JC: Temporary closure of the open abdomen: a systematic review on delayed primary fascial closure in patients with an open abdomen. World J Surg 2009, 33:199-207.

8. Bee TK, Croce MA, Magnotti LJ, Zarzaur BL, Maish GO, Minard G, Schroeppel TJ, Fabian TC: Temporary abdominal closure techniques: a prospective randomized trial comparing polyglactin 910 mesh and vacuum-assisted closure. J Trauma 2008, 65:337-344.

9. Stevens P: Vacuum-assisted closure of laparostomy wounds: a critical review of the literature. Int Wound J 2009, 6:259-266.

10. Björck M, Bruhin A, Cheatham M, Hinck D, Kaplan M, Manca G, Wild T, Windsor A: Classification--important step to improve management of patients with an open abdomen. World J Surg 2009, 33:1154-1157.

11. Petersson U, Acosta S, Bjorck M: Vacuum-assisted wound closure and meshmediated fascial traction - a novel technique for late closure of the open abdomen. World J Surg 2007, 31:2133-2137.

12. De Waele JJ, Hoste EA, Malbrain ML: Decompressive laparotomy for abdominal compartment syndrome--a critical analysis. Crit Care 2006, 10:R51.

doi:10.1186/cc8207

Cite this article as: Mentula P, Leppäniemi A: Prophylactic open abdomen in patients with postoperative intra-abdominal hypertension. Critical Care 2010, 14:111. 